\title{
Eficacia de la espironolactona en el tratamiento del acné vulgar. Una revisión sistemática
}

\author{
Efficacy of spironolactone in treatment of acne vulgaris. A systematic review \\ Ana Laura Pichardo-Ávalos ${ }^{a}$, Mario I. Ortiz ${ }^{b}$, Raquel Cariño-Cortés ${ }^{c}$
}

\begin{abstract}
:
Objective: To analyze the efficacy of spironolactone therapy in the treatment of acne vulgaris reported from 1980 to March 2021. Methods: A search was carried out in the following databases: Scopus, PubMed, Embase, Science direct, Cochrane Library and Google Scholar, those articles that reported the follow-up of patients with Acne Vulgaris treated with spironolactone were selected. Methodological quality was assessed by three reviewers using the Mixed Methods Appraisal Tool (MMAT), version 2018. Results: Fifteen articles with accompanying or comparative treatments or both and five articles with spironolactone monotherapy that were not complemented or compared were selected. Variable efficacy was reported in the 20 articles that were selected. The five adverse reactions most common in the studies analyzed were: menstrual disturbances, hyperkalemia, localized pigmentation at the treatment site, dizziness and fatigue. Conclusions: Consider that even with the diversity of types of studies, a large percentage of acne reduction is reported, therefore, the evidence indicates that the treatment could be effective under controlled conditions.
\end{abstract}

Keywords:

Acne vulgaris, spironolactone, acne treatment, hirsutism.

\section{Resumen:}

Objetivo: Analizar la eficacia de la terapia con espironolactona en el tratamiento del acné vulgar reportada desde 1980 hasta Marzo del 2021. Métodos: Se realizó una búsqueda en las siguientes bases de datos: Scopus, PubMed, Embase, Science direct, Cochrane Library y Google Scholar, se seleccionaron aquellos artículos que reportaron el seguimiento de pacientes con Acné Vulgaris tratados con espironolactona. Tres revisores evaluaron la calidad metodológica mediante la "Mixed Methods Appraisal Tool" (MMAT), versión 2018. Resultados: Se seleccionaron 15 artículos con tratamientos acompañantes, comparativos o ambos y cinco artículos con monoterapia de espironolactona que no se complementaban ni comparaban. Se reportó una eficacia variable en los 20 artículos que fueron seleccionados. Las cinco reacciones adversas con mayor frecuencia en los estudios analizados fueron: alteraciones menstruales, hiperkalemia, pigmentación localizada en el sitio del tratamiento, mareos y fatiga. Conclusiones: Considerar que aún con la diversidad de tipos de estudios, se reporta un gran porcentaje de disminución del acné, por lo tanto, las evidencias indican que el tratamiento podría ser eficaz bajo condiciones controladas.

\section{Palabras Clave:}

Acné vulgar, espironolactona, tratamiento del acné, hirsutismo.

\section{Introducción}

El acné vulgar (AV) es una enfermedad dermatológica crónica que compromete la unidad pilosebácea, ${ }^{1}$ siendo una patología de difícil tratamiento debido a la etiología multifactorial y los múltiples fracasos terapéuticos..$^{1-6} \mathrm{EI}$ $\mathrm{AV}$ es una nosología dermatológica compleja, teniendo como problema fundamental su alta prevalencia e importante impacto biopsicosocial. ${ }^{1-8} \mathrm{El} \mathrm{AV}$ se asocia con timidez, autoestima baja, ansiedad en la interacción social, insatisfacción con la apariencia y un deterioro general de la calidad de vida de los pacientes. ${ }^{6,8,9} \mathrm{La}$ prevalencia del acné varía, con estimaciones que oscilan entre el 35 y el $100 \%$ en adolescentes. ${ }^{1}$ En un estudio de

\footnotetext{
a Universidad Autónoma del Estado de Hidalgo, https://orcid.org/0000-0003-4118-5374, Email: alpa-med@ @otmail.com

b Autor de Correspondencia, Universidad Autónoma del Estado de Hidalgo, https://orcid.org/ 0000-0003-1047-6304, Email: mario_i_ortiz@hotmail.com

c Universidad Autónoma del Estado de Hidalgo, https://orcid.org/ 0000-0003-4776-3534, Email: raquelcarcortes@gmail.com
} 
1,013 participantes de 20 años o más, el $73.3 \%(n=744)$ informó haber tenido AV alguna vez, después de la adolescencia ${ }^{10}$. En el mismo estudio se encontró mayor prevalencia de $\mathrm{AV}$ en mujeres versus hombres en todos los grupos etarios (>19 años). ${ }^{10}$ Aunque la colonización temprana con Cutibacterium acnes (anteriormente Propionibacterium acnes) y la edad pueden jugar un papel en la enfermedad, no está claro exactamente qué desencadena el acné y cómo el tratamiento afecta el curso de la enfermedad. ${ }^{11}$ Factores como la dieta han sido implicados, pero no probados, así como factores de exposición al sol y el uso de cosméticos a una edad temprana ${ }^{8-11} \mathrm{El}$ acné ocurre a través de la interacción de cuatro factores principales: 1) exceso de producción de sebo, 2) obstrucción folicular con sebo y queratinocitos, 3) colonización folicular por $C$. acnés (un anaeróbico humano), y 4) liberación de múltiples mediadores inflamatorios. ${ }^{11}$ El acné se puede clasificar en: 1) No inflamatorio: caracterizado por comedones, e 2) inflamatorio: caracterizado por pápulas, pústulas, nódulos y quistes. ${ }^{11}$ En la patogenia del acné, las hormonas andrógenas juegan un papel crucial. ${ }^{1,4}$ Los dos requisitos para la actividad androgénica a nivel de unidad pilosebácea son: 1) la presencia de andrógenos en forma de testosterona o dihidrotestosterona; y 2) receptores de andrógenos funcionales. ${ }^{12}$

El tratamiento óptimo ha sido uno de los problemas siempre latentes en el manejo del acné, lo que lleva a los médicos a no siempre brindar tratamientos efectivos, que, aunque en ocasiones reducen los síntomas (datos clínicos cutáneos inflamatorios y no inflamatorios), la enfermedad no siempre se erradica. ${ }^{12}$ Para el AV se han utilizado múltiples tratamientos, tratando de atacar el origen de esta condición, como: retinoides, peróxido de benzoilo, antibióticos (doxiciclina, minociclina, tetraciclina, macrólidos, eritromicina, azitromicina, trimetoprima), tratamientos hormonales (anticonceptivos orales, antiandrógeno, corticoesteroides), herbales, dietético, antioxidantes y probióticos. ${ }^{11}$ Recientemente, se ha implementado la combinación de algunas de las terapias descritas anteriormente acompañadas de terapias de luz en diferentes longitudes de onda ${ }^{13}$. El tratamiento del acné depende de su gravedad, de acuerdo con las pautas de tratamiento del acné, en un grado leve, el tratamiento será tópico a base de terapia con antibióticos o retinoides. ${ }^{11-14}$ En un grado moderado, el tratamiento será tópico con antibiótico o retinoide más peróxido de benzoilo. ${ }^{14,15}$ Finalmente, en grado severo, el tratamiento será antibioterapia oral más peróxido de benzoilo más antiandrógeno o isotretinoína. ${ }^{14,15} \mathrm{En}$ cuanto a la terapia antiandrogénica, se han utilizado varios fármacos con diferente eficacia y seguridad como flutamida, ciproterona, espironolactona y la combinación oral con anticonceptivos. ${ }^{11-15}$ La espironolactona es un antagonista sintético de los receptores de mineralocorticoides y aldosterona, ${ }^{16}$ se usa principalmente como diurético para el tratamiento de la hipertensión, el hiperaldosteronismo y la insuficiencia cardíaca. ${ }^{16}$ La espironolactona se usa fuera de su posología aprobada, por sus propiedades antiandrogénicas para el tratamiento del AV; compite con la testosterona y la dihidrotestosterona por unirse al receptor de andrógenos. ${ }^{16}$ Los efectos sistémicos de la espironolactona sobre la síntesis suprarrenal de precursores de andrógenos también pueden contribuir a su eficacia clínica, aunque a dosis terapéuticas esto puede ser poco probable. ${ }^{16,17} \mathrm{La}$ espironolactona conlleva un riesgo de hiperkalemia e hiponatremia porque es un diurético ahorrador de potasio. ${ }^{16-18}$ Los estudios en personas sanas encontraron un bajo riesgo de hiperkalemia clínicamente significativa y en general, no requirieron seguimiento. ${ }^{11}$ Debido a la complejidad del tratamiento antiandrogénico del acné y que sus resultados no siempre reproducibles, se decidió realizar esta revisión para objetivar la eficacia de la espironolactona en esta patología.

\section{Material y Métodos}

\section{Estrategia de búsqueda}

Se realizó una revisión sistemática de la literatura científica publicada en el área de dermatología sobre el tema terapéutico del $\mathrm{AV}$ ante el uso de espironolactona. Esta revisión sistemática se realizó de acuerdo la guía para revisiones sistemáticas y metaanálisis. ${ }^{19}$ Se realizaron búsquedas en las bases de datos de "Scopus, PubMed, Embase, Science direct, Cochrane Library y Google Scholar" en busca de estudios publicados sobre el tratamiento del AV con espironolactona. Las palabras clave de búsqueda fueron: [(espironolactona y acné o acné y espironolactona) o (tratamiento del acné)]. La búsqueda se realizó del 4 de enero al 31 de marzo de 2021. Algunos otros estudios se obtuvieron mediante una búsqueda manual en Google y la selección de referencias de artículos relevantes. El protocolo del estudio fue elaborado, registrado y publicado en la base de datos internacional de revisiones sistemáticas registradas prospectivamente en salud y atención social (PROSPERO), con el registro CRD42021247491. Los criterios de inclusión de esta revisión sistemática fueron: 1) Manuscritos publicados desde el 1 de enero de 1980 hasta el 31 de marzo de 2021, 2) Texto completo en cualquier idioma, 3) Artículos que trataran sobre pacientes con $\mathrm{AV}$ y tratamiento con espironolactona, 4) Que tuvieran cualquier número de participantes, 5) De todos los grupos de edad, 6) Cualquier sexo y 7) 
Espironolactona como terapia base, en cualquiera de sus presentaciones.

Los criterios de exclusión fueron: 1) Manuscritos con fines comerciales y 2) Estudios de investigación básica. Se realizó una búsqueda exhaustiva en la base de datos de estudios originales sobre el tratamiento del AV con espironolactona. Un diagrama de flujo de PRISMA, (Figura 1) muestra el proceso involucrado en la obtención de estudios elegibles.

\section{Selección de artículos y evaluación de la calidad metodológica}

La selección de estudios se realizó según los criterios de elegibilidad. Tres revisores independientes evaluaron los títulos y los resúmenes. Se utilizó el marco de población, intervención, comparación y resultado (PICO por las siglas del nombre en inglés: "Population, Intervention, Comparation, Outcome") para determinar la elegibilidad de los estudios. Después de eso, fueron evaluados por completo al analizar su contenido. La evaluación de la calidad se realizó mediante la "Mixed Methods Appraisal Tool" (MMAT), versión 2018 en donde los puntajes de calidad total oscilaron entre 60 y $100 \%$.

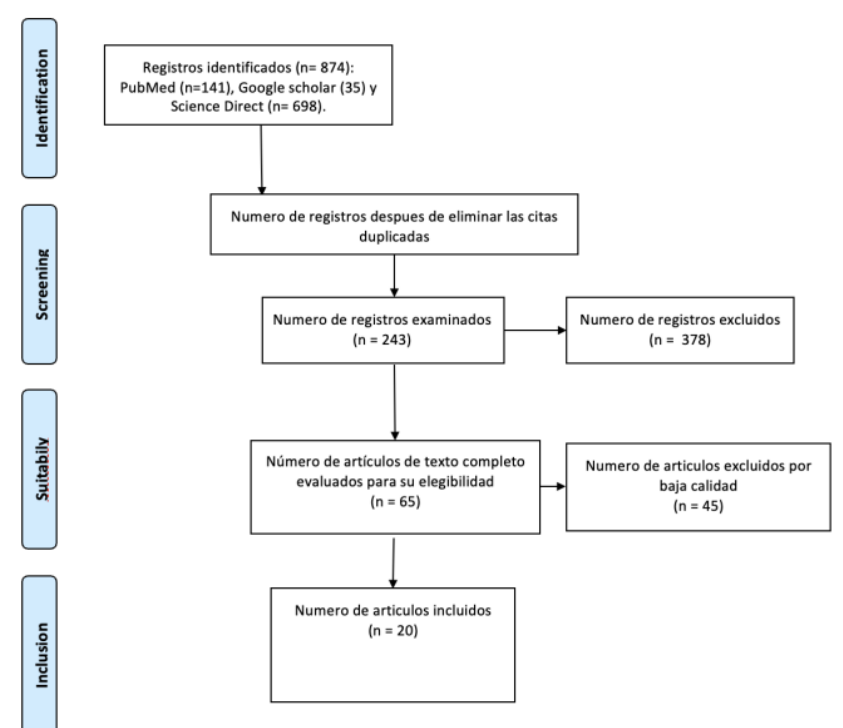

Figura 1. Diagrama de flujo PRISMA en cuatro niveles: En apoyo, este estudio incluye las pautas de elementos de notificación preferidos para revisiones sistemáticas y meta-análisis (PRISMA).

\section{Recolección y análisis de datos}

Los manuscritos fueron revisados sistemáticamente según el tratamiento del AV con espironolactona. Los datos se extrajeron de los estudios después de una extensa evaluación y revisión. Veinte manuscritos cumplieron los criterios de inclusión y fueron seleccionados para la revisión sistemática (Figura 1). La extracción de los resultados de los manuscritos fue realizada por tres revisores. Las variables de interés para cada estudio se organizaron en hojas de cálculo estandarizadas y se clasificaron según las siguientes características: autores, tipo de tratamiento suministrado, efectividad demostrada, datos de eficacia y reacciones adversas. Los resultados se organizaron en 5 tablas diferentes. Se analizaron resultados tanto cualitativos como cuantitativos.

\section{Características de las intervenciones}

Las intervenciones terapéuticas de terapia con espironolactona confirmaron resultados consistentes en los 20 estudios analizados, se incluyeron artículos con varios tipos de terapia en los que se integraron en 2 grupos. El primer grupo corresponde a los artículos cuyo tratamiento con espironolactona tiene combinaciones terapéuticas y/o la espironolactona es comparada con otro fármaco (Tabla 1). El segundo grupo pertenece a los artículos cuyo tratamiento fue único con espironolactona y no se combinó ni comparó su efectividad con otros fármacos (Tabla 2). Del mismo modo observamos que de manera independiente al tipo de terapéutica brindada las combinaciones presentaron diversos grados de efectividad reportada en los artículos. Así, para el grupo de terapia combinada y/o comparada con otros fármacos se recabaron los grados de efectividad en escaso, moderado y significativo (según el porcentaje reportado en cada artículo se estableció un 30\% o menos para resultado "escaso", 31-60\% para "moderado" y >60\% para "significativo" (Tabla 3). De igual manera se graduó con resultado escaso, moderado y significativo con los mismos porcentajes reportados para el grupo de monoterapia (Tabla 4).

\section{Resultados}

De un total de 243 artículos, 20 artículos cumplieron los criterios de inclusión y fueron seleccionados para la revisión sistemática (Figura 1), de los cuales 15 utilizaron tratamientos acompañantes o comparativos además de la espironolactona y los otros 5 únicamente utilizaron espironolactona como monoterapia. De los 20 artículos incluidos 9 fueron estudios observacionales retrospectivos y los otros 11 fueron estudios controlados aleatorizados. La población total referida en los 20 artículos fue de 44,592 pacientes (algunos de ellos únicamente fueron reportados por sus registros médicos). Del total anterior, solo se reportó el sexo de 40,352 (90.5\%) pacientes. De estos últimos, 40,289 (99.8\%) pacientes fueron femeninos y 62 (0.15\%) masculinos. De los 44,592 pacientes inicialmente registrados, solo terminaron los respectivos protocolos 40,289 , es decir, solo el $90.3 \%$ (debido a eliminación o abandono de los pacientes). De 44,592 pacientes que se 
reportaron, solo $8,981(20.1 \%)$ fueron tratados con espironolactona. El rango de edad de los pacientes reportados oscila de 12 - 66 años, con un promedio ponderado de 23 años.

Tabla 1. Análisis de tratamiento con espironolactona para estudios comparativos y/o que utilizaron combinaciones.

\begin{tabular}{|c|c|c|c|}
\hline Autor & Fármaco en combinación & Tratamiento en comparación & $\begin{array}{l}\text { Dosis de } \\
\text { espironolactona } \\
\text { usada }\end{array}$ \\
\hline $\begin{array}{l}\text { Goodfellow et } \\
\text { al., } 1984^{20}\end{array}$ & Ninguno & Placebo & $\begin{array}{l}50,100,150 \text { o } \\
200 \mathrm{mg} / \text { día }\end{array}$ \\
\hline $\begin{array}{l}\text { Afzali et al., } \\
2012^{21}\end{array}$ & Ninguno & Placebo & $\begin{array}{l}\text { Aplicación dos } \\
\text { veces día }\end{array}$ \\
\hline $\begin{array}{l}\text { Barbieri et al., } \\
2018^{22}\end{array}$ & Ninguno & Tetraciclinas & No mencionado \\
\hline $\begin{array}{l}\text { Krunic et al., } \\
2008^{23}\end{array}$ & Etinilestradiol y drospirenona. & Ninguno & 100 mg / día \\
\hline $\begin{array}{l}\text { Muhlemann et } \\
\text { al., } 1986^{24}\end{array}$ & $\begin{array}{l}\text { Anticonceptivos (en } 6 \text { de las } 21 \\
\text { pacientes). }\end{array}$ & Placebo & 200 mg / día \\
\hline $\begin{array}{l}\text { Patiyasikunt } \\
\text { et al., } 2020^{25}\end{array}$ & Peróxido de benzoilo & Placebo & 25 o 50 mg /día \\
\hline $\begin{array}{l}\text { Cusan et al., } \\
1994^{26}\end{array}$ & Anticonceptivos & Flutamida & $\begin{array}{l}50 \text { mg / } 2 \text { veces al } \\
\text { día }\end{array}$ \\
\hline $\begin{array}{l}\text { Kriplani et al., } \\
2009^{27}\end{array}$ & $\begin{array}{l}\text { Etinilestradiol } 35 \mu \mathrm{g} \text { y acetato de } \\
\text { ciproterona } 2 \mathrm{mg}\end{array}$ & $\begin{array}{l}\text { Etinilestradiol } 35 \mu \mathrm{g} \text { y acetato de } \\
\text { ciproterona } 2 \mathrm{mg} \text { más finasterida } 5 \\
\mathrm{mg}\end{array}$ & 100 mg / día \\
\hline $\begin{array}{l}\text { Park et al., } \\
2018^{28}\end{array}$ & Anticonceptivos orales y antibióticos & $\begin{array}{l}\text { Anticonceptivos orales, antibióticos y } \\
\text { placebo }\end{array}$ & 25 - 300 mg / día \\
\hline $\begin{array}{l}\text { Charny et al., } \\
2017^{29}\end{array}$ & Anticonceptivos orales y antibióticos & Anticonceptivos orales y antibióticos & $\begin{array}{l}100-200 \text { mg / } \\
\text { día }\end{array}$ \\
\hline Shaw, $2000^{30}$ & Anticonceptivos y antibióticos & $\begin{array}{l}\text { Combinación de espironolactona más } \\
\text { antibióticos o más anticonceptivos }\end{array}$ & $\begin{array}{l}100-200 \mathrm{mg} / \\
\text { día }\end{array}$ \\
\hline $\begin{array}{l}\text { Roberts et al., } \\
2021^{31}\end{array}$ & Antibióticos y anticonceptivos orales & $\begin{array}{l}\text { Combinación de espironolactona más } \\
\text { antibióticos o espironolactona más } \\
\text { anticonceptivos }\end{array}$ & 100 mg / día \\
\hline $\begin{array}{l}\text { Vijayendran } \\
\text { et al., } 2018^{32}\end{array}$ & Clindamicina & Isotretinoína más clindamicina & 50 mg / día \\
\hline $\begin{array}{l}\text { Roberts et al., } \\
2020^{33}\end{array}$ & Antibióticos y anticonceptivos orales & $\begin{array}{l}\text { Combinación de espironolactona más } \\
\text { antibióticos o más anticonceptivos } \\
\text { orales }\end{array}$ & 100 mg / día \\
\hline $\begin{array}{l}\text { Turowski y } \\
\text { James, } \\
2007^{34}\end{array}$ & $\begin{array}{l}\text { Amoxicilina y trimetoprima- } \\
\text { sulfametoxazol }\end{array}$ & $\begin{array}{l}\text { Combinación de espironolactona con } \\
\text { amoxicilina o combinación con } \\
\text { trimetoprima - sulfametoxazol. }\end{array}$ & 50 - 200 mg / día \\
\hline
\end{tabular}

Tabla 2. Análisis de tratamiento con espironolactona para estudios no comparativos.

\begin{tabular}{|l|c|l|}
\hline Autor & Vía de administración & \multicolumn{1}{|c|}{ Dosis utilizada } \\
\hline Sato et al., $2006^{35}$ & Oral & $200 \mathrm{mg} /$ día \\
\hline Isvy-Joubert et al., $2017^{36}$ & Oral & $25-150 \mathrm{mg} /$ día \\
\hline Yemisci et al., $2005^{37}$ & Oral & $50 \mathrm{mg}$ dos veces al día \\
\hline Grandhi y Alikhan, $2017^{38}$ & Oral & $50-100 \mathrm{mg} /$ día \\
\hline Cortez de Castro et al., $1986^{39}$ & Oral & $100 \mathrm{mg} /$ día \\
\hline
\end{tabular}

Tabla 3. Mejoría en el acné para estudios comparativos

\begin{tabular}{|l|l|l|c|c|c|}
\hline Artículo & Fármaco acompañante & Fármaco comparativo & Escaso & Moderado & Significativo \\
\hline $\begin{array}{l}\text { Goodfellow } \\
\text { et al., }\end{array}$ & Ninguno & Placebo & & & X \\
$1984^{20}$ & & & & & \\
\hline
\end{tabular}




\begin{tabular}{|c|c|c|c|c|c|}
\hline $\begin{array}{l}\text { Afzali et al., } \\
2012^{21}\end{array}$ & Ninguno & Placebo & $\mathrm{X}$ & & \\
\hline $\begin{array}{l}\text { Barbieri et } \\
\text { al., } 2018^{22}\end{array}$ & Ninguno & Tetraciclinas & & & $\mathrm{X}$ \\
\hline $\begin{array}{l}\text { Krunic et al., } \\
2008^{23}\end{array}$ & Etinilestradiol y drospirenona & Ninguno & & & $\mathrm{X}$ \\
\hline $\begin{array}{l}\text { Muhlemann } \\
\text { et al., } 1986^{24}\end{array}$ & $\begin{array}{l}\text { Anticonceptivos (en } 6 \text { de las } 21 \\
\text { pacientes). }\end{array}$ & Placebo & & & $\mathrm{X}$ \\
\hline $\begin{array}{l}\text { Patiyasikunt } \\
\text { et al., } 2020^{25}\end{array}$ & Peróxido de benzoilo al 2.5\% & Placebo & & $\mathrm{X}$ & \\
\hline $\begin{array}{l}\text { Cusan et al., } \\
1994^{26}\end{array}$ & Anticonceptivos & Flutamida & & $\mathrm{X}$ & \\
\hline $\begin{array}{l}\text { Kriplani et } \\
\text { al., } 2009^{27}\end{array}$ & $\begin{array}{l}\text { Etinilestradiol } 35 \mu \mathrm{g} \text { y acetato de } \\
\text { ciproterona } 2 \mathrm{mg}\end{array}$ & $\begin{array}{l}\text { Etinilestradiol } 35 \mu \mathrm{g} \text { y acetato de } \\
\text { ciproterona } 2 \mathrm{mg} \text { más finasterida }\end{array}$ & & $X$ & \\
\hline $\begin{array}{l}\text { Park et al., } \\
2018^{28}\end{array}$ & $\begin{array}{l}\text { Anticonceptivos orales y } \\
\text { antibióticos }\end{array}$ & $\begin{array}{l}\text { Anticonceptivos orales, } \\
\text { antibióticos y placebo. }\end{array}$ & & & $\mathrm{X}$ \\
\hline $\begin{array}{l}\text { Charny et } \\
\text { al., } 2017^{29}\end{array}$ & $\begin{array}{l}\text { Anticonceptivos orales y } \\
\text { antibióticos }\end{array}$ & $\begin{array}{l}\text { Anticonceptivos orales y } \\
\text { antibióticos }\end{array}$ & & & $\mathrm{X}$ \\
\hline $\begin{array}{l}\text { Shaw, } \\
2000^{30}\end{array}$ & Anticonceptivos y antibióticos & $\begin{array}{l}\text { Combinación de espironolactona } \\
\text { más antibióticos o más } \\
\text { anticonceptivos }\end{array}$ & & $\mathrm{X}$ & \\
\hline $\begin{array}{l}\text { Roberts et } \\
\text { al., } 2021^{31}\end{array}$ & $\begin{array}{l}\text { Antibióticos y anticonceptivos } \\
\text { orales }\end{array}$ & $\begin{array}{l}\text { Combinación de espironolactona } \\
\text { más antibióticos o } \\
\text { espironolactona más } \\
\text { anticonceptivos }\end{array}$ & & $\mathrm{X}$ & \\
\hline $\begin{array}{l}\text { Vijayendran } \\
\text { et al., } \\
2018^{32}\end{array}$ & Clindamicina & Isotretinoína más clindamicina & $\mathrm{X}$ & & \\
\hline $\begin{array}{l}\text { Roberts et } \\
\text { al., } 2020^{33}\end{array}$ & $\begin{array}{l}\text { Antibióticos y anticonceptivos } \\
\text { orales }\end{array}$ & $\begin{array}{l}\text { Combinación de espironolactona } \\
\text { más antibióticos o más } \\
\text { anticonceptivos orales }\end{array}$ & & & $\mathrm{X}$ \\
\hline $\begin{array}{l}\text { Turowski y } \\
\text { James, } \\
2007^{34}\end{array}$ & $\begin{array}{l}\text { Amoxicilina y trimetoprima- } \\
\text { sulfametoxazol }\end{array}$ & $\begin{array}{l}\text { Combinación de espironolactona } \\
\text { con amoxicilina o combinación } \\
\text { con trimetoprima - } \\
\text { sulfametoxazol y monoterapia. }\end{array}$ & & & $\mathrm{X}$ \\
\hline
\end{tabular}

Tabla 4. Mejoría en el acné para estudios no comparativos.

\begin{tabular}{|l|l|l|c|}
\hline Artículo & Escaso & Moderado & Significativo \\
\hline Sato et al., $2006^{35}$ & & & $\mathrm{X}$ \\
\hline Isvy-Joubert et al., $2017^{36}$ & & & $\mathrm{X}$ \\
\hline Yemisci et al., $2005^{37}$ & & & $\mathrm{X}$ \\
\hline Grandhi y Alikhan, $2017^{38}$ & & & $\mathrm{X}$ \\
\hline Cortez de Castro et al., $1986^{39}$ & & $\mathrm{X}$ & \\
\hline
\end{tabular}

Tabla 5. Reacciones adversas de la espironolactona

\begin{tabular}{|c|c|c|c|c|}
\hline Efecto(s) adverso(s) & $\begin{array}{c}\text { Participantes } \\
\text { expuestos }\end{array}$ & $\begin{array}{l}\text { Participantes } \\
\text { que la presentó }\end{array}$ & $\%$ & Referencias \\
\hline Alteraciones menstruales & 1334 & 255 & 19.1 & 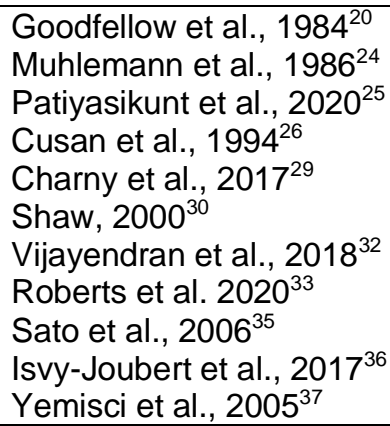 \\
\hline
\end{tabular}




\begin{tabular}{|c|c|c|c|c|}
\hline & & & & Grandhi y Alikha, $2017^{38}$ \\
\hline Hiperkalemia & 80 & 10 & 12.5 & Shaw, $2000^{30}$ \\
\hline $\begin{array}{l}\text { Pigmentación localizada en el } \\
\text { sitio de tratamiento }\end{array}$ & 36 & 4 & 11.1 & Afzali et al., $2012^{21}$ \\
\hline Mareos & 672 & 65 & 9.6 & $\begin{array}{l}\text { Muhlemann et al., } 1986^{24} \\
\text { Patiyasikunt et al., } 2020^{25} \\
\text { Cusan et al., } 1994^{26} \\
\text { Charny et al., } 2017^{29} \\
\text { Roberts et al., } 2020^{33} \\
\text { Isvy-Joubert et al., } 2017^{36} \\
\text { Yemisci et al., } 2005^{37}\end{array}$ \\
\hline Fatiga & 613 & 56 & 9.1 & $\begin{array}{l}\text { Charny et al., } 2017^{29} \\
\text { Shaw, } 2000^{30} \\
\text { Roberts et al., } 2020^{33} \\
\text { Yemisci et al., } 2005^{37}\end{array}$ \\
\hline Descamación & 36 & 3 & 8.3 & Afzali et al., $2012^{21}$ \\
\hline Sensibilidad mamaria & 388 & 23 & 5.9 & $\begin{array}{l}\text { Patiyasikunt et al., } 2020^{25} \\
\text { Cusan et al., } 1994^{26} \\
\text { Charny et al., } 2017^{29} \\
\text { Shaw, } 2000^{30} \\
\text { Roberts et al., } 2021^{31} \\
\text { Isvy-Joubert et al., } 2017^{36}\end{array}$ \\
\hline $\begin{array}{l}\text { Ardor en el sitio de } \\
\text { tratamiento }\end{array}$ & 36 & 2 & 5.5 & Afzali et al., $2012^{21}$ \\
\hline Náuseas & 201 & 10 & 4.9 & $\begin{array}{l}\text { Muhlemann et al., } 1986^{24} \\
\text { Kriplani et al., } 2009^{27} \\
\text { Shaw, } 2000^{30} \\
\text { Yemisci et al., } 2005^{37} \\
\text { Cortez de Castro et al., } \\
1986^{39}\end{array}$ \\
\hline Diuresis aumentada & 266 & 12 & 4.5 & $\begin{array}{l}\text { Goodfellow et al., } 1984^{20} \\
\text { Charny et al., } 2017^{29} \\
\text { Shaw, } 2000^{30} \\
\text { Yemisci et al., } 2005^{37} \\
\text { Cortez de Castro et al., } \\
1986^{39}\end{array}$ \\
\hline Calambres / Tetania & 70 & 3 & 4.2 & Isvy-Joubert et al., $2017^{36}$ \\
\hline Cefalea & 122 & 5 & 4.1 & $\begin{array}{l}\text { Roberts et al., } 2021^{31} \\
\text { Vijayendran et al., } 2018^{32} \\
\text { Cortez de Castro et al., } \\
1986^{39}\end{array}$ \\
\hline Erupción cutánea & 80 & 3 & 3.8 & Roberts et al., $2021^{31}$ \\
\hline Diarrea & 80 & 3 & 3.8 & Roberts et al., $2021^{31}$ \\
\hline Hipotensión arterial & 150 & 4 & 2.6 & $\begin{array}{l}\text { Isvy-Joubert et al., } 2017^{36} \\
\text { Shaw, } 2000^{30}\end{array}$ \\
\hline Melasma & 80 & 2 & 2.5 & Shaw, $2000^{30}$ \\
\hline Aumento de peso & 122 & 3 & 2.5 & $\begin{array}{l}\text { Charny et al., } 2017^{29} \\
\text { Cortez de Castro et al., } \\
1986^{39}\end{array}$ \\
\hline Edema en extremidades & 176 & 4 & 2.3 & $\begin{array}{l}\text { Kriplani et al., } 2009^{27} \\
\text { Sato et al., } 2006^{35}\end{array}$ \\
\hline Somnolencia & 110 & 2 & 1.8 & Charny et al., $2017^{29}$ \\
\hline Piernas inquietas & 110 & 2 & 1.8 & Charny et al., $2017^{29}$ \\
\hline Pérdida de peso & 180 & 3 & 1.6 & $\begin{array}{l}\text { Charny et al., } 2017^{29} \\
\text { Isvy-Joubert et al., } 2017^{36}\end{array}$ \\
\hline Hipertensión & 60 & 1 & 1.6 & Kriplani et al., $2009^{27}$ \\
\hline Depresión & 60 & 1 & 1.6 & Kriplani et al., $2009^{27}$ \\
\hline Xerosis & 80 & 1 & 1.25 & Shaw, $2000^{30}$ \\
\hline Disminución libido & 190 & 2 & 1.05 & $\begin{array}{l}\text { Charny et al., } 2017^{29} \\
\text { Shaw, } 2000^{30}\end{array}$ \\
\hline
\end{tabular}




\begin{tabular}{|c|c|c|c|c|}
\hline Deshidratación & 110 & 1 & 0.90 & Charny et al., $2017^{29}$ \\
\hline Caída de cabello & 110 & 1 & 0.90 & Charny et al., $2017^{29}$ \\
\hline Prurito & 110 & 1 & 0.90 & Charny et al., $2017^{29}$ \\
\hline Fotosensibilidad & 110 & 1 & 0.90 & Charny et al., $2017^{29}$ \\
\hline Ansiedad & 110 & 1 & 0.90 & Charny et al., $2017^{29}$ \\
\hline Taquicardia & 110 & 1 & 0.90 & Charny et al., $2017^{29}$ \\
\hline Sabor metálico & 110 & 1 & 0.90 & Charny et al., $2017^{29}$ \\
\hline Dolor abdominal & 110 & 1 & 0.90 & Charny et al., $2017^{29}$ \\
\hline
\end{tabular}

\section{Efectos de las intervenciones}

Todos los estudios incluidos con intervenciones basadas en espironolactona ofrecieron resultados positivos en pacientes con acné en sus diferentes grados clínicos. Aunque la efectividad por cada grupo fue variable, en los 15 manuscritos del grupo de terapia combinada y/o comparativa, solo dos (13.3\%) artículos mostraron eficacia escasa ${ }^{21,32}$, seis estudios $(40 \%)$ describieron la eficacia como moderada ${ }^{20,25,26,27,30,31}$ mientras que siete manuscritos $(46.7 \%)$ mencionaron una eficacia significativa 22-24,28,29,33,34 (Tabla 3). De los estudios pertenecientes al grupo de monoterapia, en un manuscrito $(20 \%)$ se concluyó una eficacia moderada ${ }^{39}$ y en cuatro $(80 \%)$ artículos se encontró una eficacia significativa ${ }^{35-38}$ (Tabla 4).

\section{Reacciones adversas}

Dieciocho de los 20 artículos que fueron incluidos en la revisión reportaron diversas reacciones adversas. Las alteraciones menstruales fueron las más comunes, ${ }^{20,26,29,30-33,35-38}$ seguido por la hiperkalemia, ${ }^{30}$ pigmentación localizada en la zona en donde se utilizó la terapia tópica, ${ }^{21}$ mareos, ${ }^{24-26,29,33,36,37}$ y fatiga, ${ }^{29,30,33,37} \mathrm{Se}$ presentaron otras reacciones adversas con menos frecuencia (Tabla 5).

\section{Discusión}

El AV es una patología dermatológica compleja y con una prevalencia global considerable. ${ }^{8}$ Esta nosología afecta a hombres y mujeres, con una prevalencia aumentada en pacientes de género femenino. ${ }^{10}$ Sin embargo, el aumento anómalo o bien exacervado de andrógenos en mujeres, favorece la aparición de AV en ellas en etapas post adolescentes $\quad y \quad$ premenopáusicas. ${ }^{18,40}$ Desafortunadamente, múltiples tratamientos han sido utilizados para el AV sin lograr una completa eficacia. Lo anterior llevó a probar diferentes estrategias terapéuticas, incluyendo el uso de la espironolactona como una opción terapéutica en el AV de origen androgénico. ${ }^{13}$ La presente revisión analizó 20 manuscritos y en la mayoría de éstos la eficacia de espironolactona fue de moderada a significativa (Tablas 3 y 4). Lo que muestra un resultado coherente con lo descrito en otras revisiones en donde reportan una mejoría del $\mathrm{AV}$ con espironolactona de $52 \%$ a $96 \% .^{41,42}$ En este sentido, se propone a la espironolactona como una opción terapéutica para el $\mathrm{AV}$, principalmente en pacientes con las siguientes características: mujeres en etapa post adolescente a pre menopaúsica, con rasgos clínicos de hiperandrogenismo, historia previa acné con falla al tratamiento, clínica de hirsutismo o con lesiones cutáneas asociadas a altos niveles plasmáticos de andrógenos. ${ }^{3,43-45}$ Es importante considerar las reacciones adversas que pueden presentarse al consumir este fármaco. Las reacciones más comunes con el uso de la espironolactona por vía oral (100 - $500 \mathrm{mg} /$ día) como diurético en casos de hipertensión pulmonar o edema son: hiperkalemia, deshidratación, hiponatremia, síntomas gastrointestinales, molestias neurológicas, exantema y ginecomastia. ${ }^{46,47}$ En la presente revisión, los manuscritos reportaron diversas reacciones adversas, en diferente frecuencia, dependientes de la dosis y la vía de administración (Tabla 5). Las 5 reacciones adversas más comunes fueron alteraciones menstruales, hiperkalemia, pigmentación localizada en el sitio del tratamiento, mareos $y$ fatiga. Estos hallazgos concuerdan con las reacciones adversas reportadas en otros estudios en donde los efectos secundarios más comunes fueron efectos diuréticos $(29 \%)$, irregularidades menstruales $(22 \%)$ y sensibilidad en los senos (17\%) tambien fueron reportados el agrandamiento de los senos, hipotensión ortostática, hiperkalemia y reducción de la libido. ${ }^{48,49}$

Las debilidades de la presente revisión son el número de artículos incluidos, pues gran parte de los artículos encontrados tenían al AV como una patología acompañante o bien se utilizaban formulaciones distintas a la espironolactona para el tratamiento del AV. Por otro lado, dos de los artículos no mencionaron las reacciones adversas, lo que genera inconsistencias con la presente revisión y revisiones anteriores. ${ }^{41,42}$

Sin lugar a dudas se establece a la espironolactona como base porible de un efectivo tratamiento que nos puede ayudar a disminuir el riesgo de antibioticoterapia..$^{50}$

De tal manera que, para desarrollar futuras revisiones o bien estudios de este tema, se sugiere incluir un número más grande de estudios y generar flexibilidad y especificidad en el momento de establecer los criterios de búsqueda. Asimismo, incluir variantes de acné de 
diferentes etiologías, lo que permitirá al investigador notar y establecer las variantes nosológicas y de potencial blanco terapéutico.

Resumen de resultados:

1. Todos los artículos incluidos en la revisión cuentan con una calidad metodológica adecuada según el MMAT versión 2018.

2. El $100 \%$ de los artículos muestra eficacia de la espironolactona para el AV en diferentes grados.

3. La terapia combinada de otros fármacos (antibióticos y no antibióticos), en conjunto con espironolactona se muestra como favorable ante el AV de origen androgénico.

4. Las reacciones adversas de la espironolactona son variables, en su mayoría tolerables (no causan discapacidad ni ponen en peligro la vida de los pacientes), por lo que para su uso se debe evaluar la relación riesgo-beneficio.

5. Algunos artículos mostraron que la espironolactona favorece el tratamiento de otras nosologías originadas del hiperandrogenismo aparte del AV como lo son: hirsutismo y alopecia.

\section{Conclusiones}

La presente revisión encontró que los pacientes con AV de origen androgénico, que reciben terapia con espironolactona parecen evolucionar favorablemente. Debido a la heterogeneidad en los diseños, los instrumentos de medición y los tratamientos aplicados, esta conclusión debe ser considerada con fines de orientación en el tratamiento individualizado del AV.

\section{Conflicto de intereses}

Los autores declaran que no existe ningún conflicto de intereses con este artículo.

\section{Referencias}

[1] Heng AHS, Chew FT. Systematic review of the epidemiology of acne vulgaris. Sci Rep. 2020;10(1):5754.

[2] Schäfer T, Nienhaus A, Vieluf D, Berger J, Ring J. Epidemiology of acne in the general population: the risk of smoking. Br J Dermatol. 2001;145(1):100-4.

[3] Bagatin E, Timpano DL, Guadanhim LR dos S, Nogueira VMA, Terzian LR, Steiner D, et al. Acne vulgaris: prevalence and clinical forms in adolescents from São Paulo, Brazil. An Bras Dermatol. 2014;89(3):428-35.

[4] Bhate K, Williams HC. Epidemiology of acne vulgaris: Epidemiology of acne vulgaris. $\mathrm{Br} \mathrm{J}$ Dermatol. 2013;168(3):474-85.

[5] Janani S, Sureshkumar RA. Comprehensive Review on Acne, its Pathogenesis, Treatment, In-Vitro and In-Vivo Models for Induction and Evaluation Methods. Int J Pharm Sci. 2019; 10(7): 3155-77.
[6] Law MPM, Chuh AAT, Lee A, Molinari N. Acne prevalence and beyond: acne disability and its predictive factors among Chinese late adolescents in Hong Kong: Acne disability and its predictive factors among Chinese late adolescents. Clin Exp Dermatol. 2010;35(1):16-21.

[7] Agüero de Zaputovich F, Samudio M. Características clínicas y epidemiológicas de pacientes que consultan por acné. Mem Inst Investig Cienc Salud. 2015;13(1):7-16.

[8] White GM. Recent findings in the epidemiologic evidence, classification, and subtypes of acne vulgaris. J Am Acad Dermatol. 1998;39(2):34-7

[9] Krowchuk DP. Managing acne in adolescents. Pediatr Clin North Am. 2000;47(4):841-57.

[10] Collier CN, Harper JC, Cafardi JA, Cantrell WC, Wang W, Foster KW, et al. The prevalence of acne in adults 20 years and older. J Am Acad Dermatol. 2008;58(1):56-9.

[11] Zaenglein AL, Pathy AL, Schlosser BJ, Alikhan A. Guidelines of care for the management of acne vulgaris. J Am Acad Dermatol. 2016;74(5):945-73.

[12] Shaw JC. Acne: effect of hormones on pathogenesis and management. American Journal of Clinical Dermatology. Am J Clin Dermatol. 2002;3(8):571-8

[13] Strauss JS, Krowchuk DP, Leyden JJ, Lucky AW, Shalita AR, Siegfried EC, et al. Guidelines of care for acne vulgaris management. J Am Acad Dermatol. 2007;56(4):651-63.

[14] Mills OH, Kligman AM, Pochi P, Comite H. Comparing 2.5\%, $5 \%$, and $10 \%$ benzoyl peroxide on inflammatory acne vulgaris. Int J Dermatol. 1986;25(10):664-7.

[15] Leyden JJ, Hickman JG, Jarratt MT, Stewart DM, Levy SF. The efficacy and safety of a combination benzoyl peroxide/ clindamycin topical gel compared with benzoyl peroxide alone and a benzoyl peroxide/erythromycin combination product. J Cutan Med Surg. 2001; 5:37-42.

[16] Armanini D, Andrisani A, Bordin L, Sabbadin C. Spironolactone in the treatment of polycystic ovary syndrome. Expert Opinion on Pharmacotherapy. 2016;17(13):1713-5.

[17] Menard RH, Guenthner TM, Kon H, Gillette JR. Studies on the destruction of adrenal and testicular cytochrome P-450 by spironolactone. Requirement for the 7alpha-thio group and evidence for the loss of the heme and apoproteins of cytochrome P-450. J Biol Chem. 1979;254(5):1726-33.

[18] Azarchi S, Bienenfeld A, Lo Sicco K, Marchbein S, Shapiro J, Nagler AR. Androgens in women. J Am Acad Dermatol. 2019;80(6):1509-21.

[19] Moher D, Liberati A, Tetzlaff J, Altman DG, PRISMA Group. Preferred reporting items for systematic reviews and metaanalyses: the PRISMA statement. PLoS Med. 2009;6(7):e1000097.

[20] Goodfellow A, Alaghband-Zadeh J, Carter G, Cream JJ, Holland $\mathrm{S}$, Scully J, et al. Oral spironolactone improves acne vulgaris and reduces sebum excretion. Br J Dermatol. 1984;111(2):209-14.

[21] Afzali BM, Yaghoobi E, Yaghoobi R, Bagherani N, Dabbagh MA. Comparison of the efficacy of $5 \%$ topical spironolactone gel and placebo in the treatment of mild and moderate acne vulgaris: a randomized controlled trial. J Dermatolog Treat. 2012;23(1):21-5. 
[22] Barbieri JS, Choi JK, Mitra N, Margolis DJ. Frequency of treatment switching for spironolactone compared to oral tetracycline-class antibiotics for women with acne: A retrospective cohort study 2010-2016. J Drugs Dermatol. 2018;17(6):632-8

[23] Krunic A, Ciurea A, Scheman A. Efficacy and tolerance of acne treatment using both spironolactone and a combined contraceptive containing drospirenone. J Am Acad Dermatol. 2008;58(1):60-2.

[24] Muhlemann MF, Carter GD, Cream JJ, Wise P. Oral spironolactone: an effective treatment for acne vulgaris in women. Br J Dermatol. 1986;115(2):227-32.

[25] Patiyasikunt M, Chancheewa B, Asawanonda P, Noppakun N Kumtornrut C. Efficacy and tolerability of low-dose spironolactone and topical benzoyl peroxide in adult female acne: A randomized, double-blind, placebo-controlled trial. J Dermatol. 2020;47(12):1411-6.

[26] Cusan L, Dupont A, Gomez J-L, Tremblay RR, Labrie F. Comparison of flutamide and spironolactone in the treatment of hirsutism: a randomized controlled trial. Fertil Steril. 1994;61(2):281-7.

[27] Kriplani A, Thulkar J, Agrawal N, Kulshrestha V, Ammini AC, Kumar G. A comparative study of Díane-35 plus spironolactone and Díane-35 plus finasteride in cases of hirsutism and acne. Int J Endocrinol Metab. 2009; 7:235-41

[28] Park JH, Bienenfeld A, Orlow SJ, Nagler AR. The Use of Hormonal Antiandrogen Therapy in Female Patients with Acne: A 10-Year Retrospective Study. Am J Clin Dermatol. 2018;19(3):449-55.

[29] Charny JW, Choi JK, James WD. Spironolactone for the treatment of acne in women, a retrospective study of 110 patients. International Journal of Women's Dermatology. 2017;3(2):111-5.

[30] Shaw JC. Low-dose adjunctive spironolactone in the treatmen of acne in women: A retrospective analysis of 85 consecutively treated patients. J Am Acad Dermatol. 2000;43(3):498-502.

[31] Roberts EE, Nowsheen S, Davis DMR, Hand JL, Tollefson MM, Wetter DA. Use of spironolactone to treat acne in adolescent females. Pediatr Dermatol. 2021;38(1):72-6.

[32] Vijayendran N, Kale M, Jamale V, Nikam B, Hussain A, Patil SJ. Efficacy and Safety Profile of Spironolactone $50 \mathrm{mg}$ v/s Isotretinoin $10 \mathrm{mg}$ in the Treatment of Female Patients with Acne Vulgaris Grade 1-2 - A Double Blinded, Randomized Comparative Study. JMSCR. 2018;6(2):166-78.

[33] Roberts EE, Nowsheen S, Davis MDP, McEvoy MT, Newman CC, Sartori Valinotti JC, et al. Treatment of acne with spironolactone: a retrospective review of 395 adult patients at Mayo Clinic, 2007-2017. J Eur Acad Dermatol Venereol. 2020;34(9):2106-10.

[34] Turowski CB, James WD. The efficacy and safety of amoxicillin, trimethoprim-sulfamethoxazole, and spironolactone for treatment-resistant acne vulgaris. Adv Dermatol. 2007; 23:155-63.

[35] Sato K, Matsumoto D, Iizuka F, Aiba-Kojima E, Watanabe-Ono A, Suga $\mathrm{H}$, et al. Anti-androgenic therapy using oral spironolactone for acne vulgaris in Asians. Aesthetic Plast Surg. 2006;30(6):689-94.

[36] Isvy-Joubert A, Nguyen J-M, Gaultier A, Saint-Jean M, Le Moigne M, Boisrobert E, et al. Adult female acne treated with spironolactone: A retrospective data review of 70 cases. Eur J Dermatol. 2017;27(4):393-8.

[37] Yemisci A, Gorgulu A, Piskin S. Effects and side-effects of spironolactone therapy in women with acne. J Eur Acad Dermatol Venereol. 2005;19(2):163-6.

[38] Grandhi R, Alikhan A. Spironolactone for the treatment of acne: A 4-year retrospective study. Dermatology. 2017;233(2-3):1414

[39] Cortez de Castro G, Pacheco A, Naranjo H, Palacios A. Spironolactone in thetreatment of acne [Espironolactona en el tratamiento del acne]. Dermatol Venez. 1986; 24:103-105.

[40] Arora MK, Yadav A, Saini V. Role of hormones in acne vulgaris. Clin Biochem. 2011;44(13):1035-40.

[41] Layton AM, Eady EA, Whitehouse H, Del Rosso JQ, Fedorowicz Z, van Zuuren EJ. Oral spironolactone for acne vulgaris in adult females: A hybrid systematic review. Am J Clin Dermatol. 2017;18(2):169-91.

[42] Searle TN, Al-Niaimi F, Ali FR. Spironolactone in dermatology: uses in acne and beyond. Clin Exp Dermatol. 2020;45(8):98693.

[43] Lucky AW. Endocrine aspects of acne. Pediatr Clin North Am. 1983;30(3):495-9.

[44] Zouboulis C, Chen W-C, Thornton M, Qin K, Rosenfield R. Sexual hormones in human skin. Horm Metab Res. 2007;39(2):85-95

[45] Alan S, Cenesizoglu E. Effects of hyperandrogenism and high body mass index on acne severity in women. Saudi Med J. 2014;35(8):886-9.

[46] Batterink J, Stabler SN, Tejani AM, Fowkes CT. Spironolactone for hypertension. Cochrane Database Syst Rev. 2010;(8):CD008169.

[47] Carone L, Oxberry SG, Twycross R, Charlesworth S, Mihalyo M, Wilcock A. Spironolactone. J Pain Symptom Manage. 2017;53(2):288-92.

[48] Shaw JC, White LE. Long-term safety of spironolactone in acne: Results of an 8-year followup study. J Cutan Med Surg. 2002;6(6):541-5.

[49] Katsambas AD, Dessinioti C. Hormonal therapy for acne: why not as first line therapy? facts and controversies. Clin Dermatol. 2010;28(1):17-23

[50] Han JJ, Faletsky A, Barbieri JS, Mostaghimi A. New acne therapies and updates on use of spironolactone and isotretinoin: A narrative review. Dermatol Ther (Heidelb). 2021;11(1):79_91 